\title{
Nonlinear Responses and Optical Properties Modification of SK3 under Irradiation of Femtosecond Laser Pulses
}

\author{
K. JAmShidi-GhaleH* AND H. REZAPOUR \\ Department of Physics, Azarbaijan University of Tarbiat Moallem, Tabriz, Iran
}

\begin{abstract}
In this paper, the nonlinear responses and optical properties modification in SK3 glass sample are investigated. The nonlinear responses measurements were performed by using of nonlinear transmission monitoring of $200 \mathrm{fs}$ at $800 \mathrm{~nm}$ laser pulses. The optical power limiting threshold of the sample is measured to be about $40 \mathrm{~mJ} / \mathrm{cm}^{2}$. The decrease of transmitted intensity is about $63 \%$ compared to the theoretical linear transmission at $270 \mathrm{~mJ} / \mathrm{cm}^{2}$ incident pulse energy. For prediction of the experimental nonlinear behavior results, a theoretical model based on three-photon absorption and absorption of free carriers is presented. By fitting of experimental data with theoretical model, three-photon absorption coefficient and free-carrier absorption cross-section are reported. The photo-induced optical properties modification of the SK3 sample is investigated also. The modifications that appear as darkening are observed below breakdown threshold intensity. To analyses of modified region sizes, the laser pulse energy accumulation model is applied.
\end{abstract}

PACS numbers: 33.80.Wz, 78.20.Ci

\section{Introduction}

With accessible to UV laser sources, the process of transparent materials and study of their nonlinear responses become important [1-3]. On the other hand, in recent years, the development of high power IR lasers that generate femtosecond pulses permits the same ability with degree of accuracy that was previously unattainable in the IR region [4]. Materials that exhibit nonlinear optical properties have a large potential for photonic applications and it is important to characterize their behavior by estimating parameters such as the nonlinear absorption coefficients and the nonlinear refractive index. Among the materials known, a number of special glasses, crystals and ceramics have been identified as candidates for optical switching and optical power limiting [1, 2].

With development of IR femtosecond lasers, a new field in laser processing is also opened by a laser-induced modification of the optical properties inside transparent materials, preferentially optical glasses. This is referred to as nik-engineering $[5,6]$, relating the experimental technique to changes of the complex refractive index $(n+\mathrm{i} k)$. With this technique waveguides, gratings, fiber couplers, micro-holes and three-dimensional optical memories have been fabricated in glasses [7-9]. This method relies on the use of nonlinear multi-photon absorption for coupling the laser energy into a transparent material and has allowed the photowriting of three-dimensional microstructures inside bulk glass [6-12]. In spite of laser induced permanent changes, at intensities well below glass dam-

\footnotetext{
* corresponding author; e-mail: k-jamshidi@azaruniv.edu
}

age threshold, another type of modification so called color centers or darkening induces inside glass [13, 14]. Color centers form in glass when electrons are ionized to above the glass mobility threshold. Free electrons or holes are trapped by defects in the glass and new levels are formed between the forbidden bands. The trapped particles exhibit localized energy levels that typically have absorption bands in the infrared, visible, and ultraviolet regions, making glasses colorful. Color centers are easily distinguishable from permanent laser induced damage because thermal annealing at above $150^{\circ} \mathrm{C}$ bleaches the darkened regions, i.e. erases the induced absorption bands and returns the glass to its original transparent state without cracking.

In this paper, the nonlinear optical response and erasable optical properties modification of SK3 glass sample is investigated. To prediction of experimental results of nonlinear responses the theoretical model based on the simultaneous absorption of one, two and three-photon absorption is applied. Free carriers or excited states in some media (semiconductors, glasses, etc.) can lead to the nonlinear absorption and nonlinear refraction independently of the nature of their appearance. The values of three-photon absorption coefficients and free-carriers absorption cross-section are reported. To prediction of optical properties modification the pulse accumulation model is applied [15].

\section{Experimental}

In our experiment, a Ti:sapphire femtosecond pulsed laser, operating at $\lambda=800 \mathrm{~nm}$ central wavelength and 
$\tau=200$ fs pulse duration is used. The available maximum single pulse energy for the study was $450 \mu \mathrm{J}$. A shutter and an attenuator are used to control the number of laser shots and the single pulse energy, measured with an energy monitor device (LTB) located in front of the sample surface. The laser beam diameter was reduced by a quartz lens with a focal length of $500 \mathrm{~mm}$. The measured $1 / \mathrm{e}^{2}$ diameter laser beam (Gaussian intensity distribution) at the glass samples equals to $320 \mu \mathrm{m}$. The Rayleigh length is in the order of cm in this focusing alignment at $800 \mathrm{~nm}$. With this optical geometry the maximum applied peak irradiance on the glass target was $3 \times 10^{12} \mathrm{~W} \mathrm{~cm}^{-2}$, which is two orders of magnitudes lower than the glass breakdown threshold [16]. The glass sample, $2 \mathrm{~mm}$ thick SK3, was fixed on a computerized XY positioning stage for vertical and horizontal translation.

\section{Results and discussions}

The optical limiting (OL) experiments were performed by using a Ti:sapphire laser $(800 \mathrm{~nm}, 200 \mathrm{fs}, 1 \mathrm{kHz})$. The laser beam was split into two: one beam was used to monitor the incident laser energy and the other beam was focused onto the sample using a $50 \mathrm{~cm}$ focal length lens. The energies of the incident and the transmitted pulses were simultaneously measured using two large-area photodetectors connected to a fast oscilloscope. The photodetector used to monitor the transmitted beam was placed in the far-field region and the light intensity was totally collected to avoid misinterpretation of the data due to contributions of nonlinear refraction.

The results of the transmission measurements through the SK3 glass sample are plotted as a function of incident pulse energy in Fig. 1. As is clearly shown, for incident pulse energies above $40 \mu \mathrm{J}$, the transmission becomes nonlinear. A considerable decrease (by factor of 2.2) of transmitted energy is observed for $120 \mu \mathrm{J}$ applied incident pulse energies. The transmission measurements result, as depicted in Fig. 1, shows a good optical limiting effect in SK3 glass sample.

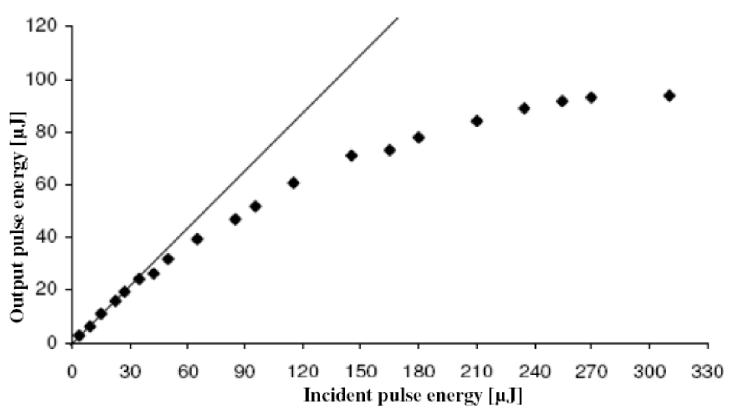

Fig. 1. The symbols show the variation in output pulse energy with input pulse energy through $2 \mathrm{~mm}$ SK3 glass sample exposed to $200 \mathrm{fs}$ laser pulses at $800 \mathrm{~nm}$. Solid line shows the $90 \%$ linear transmittance of the sample.

To characterize the nonlinear responses and optical limiting behavior of the SK3 glass sample, its nonlinear absorption is modeled. We consider a nonlinear medium of length $L$ located between the plane $z=0$ and $z=L$. The nonlinear transmission through the sample in the presence of single, two- and three-photon absorption with regarding of free-carrier absorption can be written as (for details of model see Refs. [17, 18]):

$$
T=\frac{I(L)}{I_{0}}=\frac{\mathrm{e}^{-\alpha L}}{1+\beta I_{0} L_{\mathrm{eff}}+\gamma I_{0}^{2} L_{\mathrm{eff}}^{\prime}+\eta I_{0}^{3} L_{\mathrm{eff}}^{\prime \prime}},
$$

where $L_{\text {eff }}, L_{\text {eff }}^{\prime}$ and $L_{\text {eff }}^{\prime \prime}$ are defined as:

$$
\begin{aligned}
& L_{\mathrm{eff}}=\left(1-\mathrm{e}^{-\alpha L}\right) / \alpha, \\
& L_{\mathrm{eff}}^{\prime}=\frac{\alpha}{\beta^{2} I_{0}^{2}}\left[\left(1+\beta I_{0} / \alpha\right) \ln \left(1+\beta I_{0} L_{\mathrm{eff}}\right)-\beta I_{0} L_{\mathrm{eff}}\right], \\
& L_{\mathrm{eff}}^{\prime \prime}=\frac{\alpha^{2}}{\beta^{3} I_{0}^{3}}\left[\frac{\beta I_{0} L_{\mathrm{eff}}}{1+\beta I_{0} L_{\mathrm{eff}}}\left(1+\beta I_{0} / \alpha\right)^{2}+\beta I_{0} L_{\mathrm{eff}}\right. \\
& \left.\quad-2\left(1+\beta I_{0} / \alpha\right) \ln \left(1+\beta I_{0} L_{\mathrm{eff}}\right)\right] .
\end{aligned}
$$

where $\alpha$ is effective linear absorption coefficient, $\beta$ and $\gamma$ are two and three-photon absorption coefficients respectively and $\eta$ is a constant that relates to the free-electron absorption cross section, $\sigma$. The values of $\alpha=0.72 \mathrm{~cm}^{-1}$ and $\beta=2.3 \times 10^{-12} \mathrm{~cm} \mathrm{~W}^{-1}$ are determined by $z$-scan in a separate experiment. To obtain the values of $\gamma$ and $\sigma$ the experimental results of nonlinear transmission are compared with the predictions of presented model. The solid line in Fig. 2 represents a fitting of Eq. (1) with the experimental data using the nonlinear least-square-fitting procedure (Origin) and the obtained values are: $\gamma=2.3 \times 10^{-24} \mathrm{~cm}^{3} \mathrm{~W}^{-2}$ and $\sigma=3.2 \times 10^{-18} \mathrm{~cm}^{2}$. The results are in good agreement with the other silicate glasses [18-20].

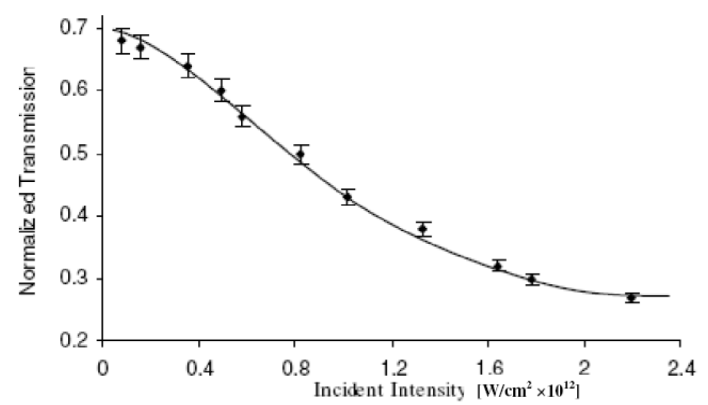

Fig. 2. The symbols show the experimental data of transmission measurement in $2 \mathrm{~mm}$ SK3 sample for $200 \mathrm{fs}$ laser pulses at $800 \mathrm{~nm}$ wavelength. The solid curve is the theoretical fit using Eq. (1) for $\alpha=$ $0.72 \mathrm{~cm}^{-1}, \beta=2.3 \times 10^{-12} \mathrm{~cm} \mathrm{~W}^{-1}, \gamma=2.3 \times$ $10^{-24} \mathrm{~cm}^{3} \mathrm{~W}^{-2}$ and $\sigma=3.2 \times 10^{-18} \mathrm{~cm}^{2}$.

To illustrate the laser fluence and shot number dependence of femtosecond laser induced darkening on SK3 glass sample, several dot fields in an arrays were generated in $2 \mathrm{~mm}$ thick sample. Figure 3 shows the optical microscope image of the visibly darkened dotes at different incident laser fluences and shot numbers. In Fig. 3 incident laser fluence varies from $0.05 \mathrm{~J} \mathrm{~cm}^{-2}$ (up) to 
$0.65 \mathrm{~J} \mathrm{~cm}^{-2}$ (down) and number of laser shots incident on a site varies from 1 (left) to 1000 (right). We must mention that the applied laser fluence levels are well below the glass breakdown threshold [16].

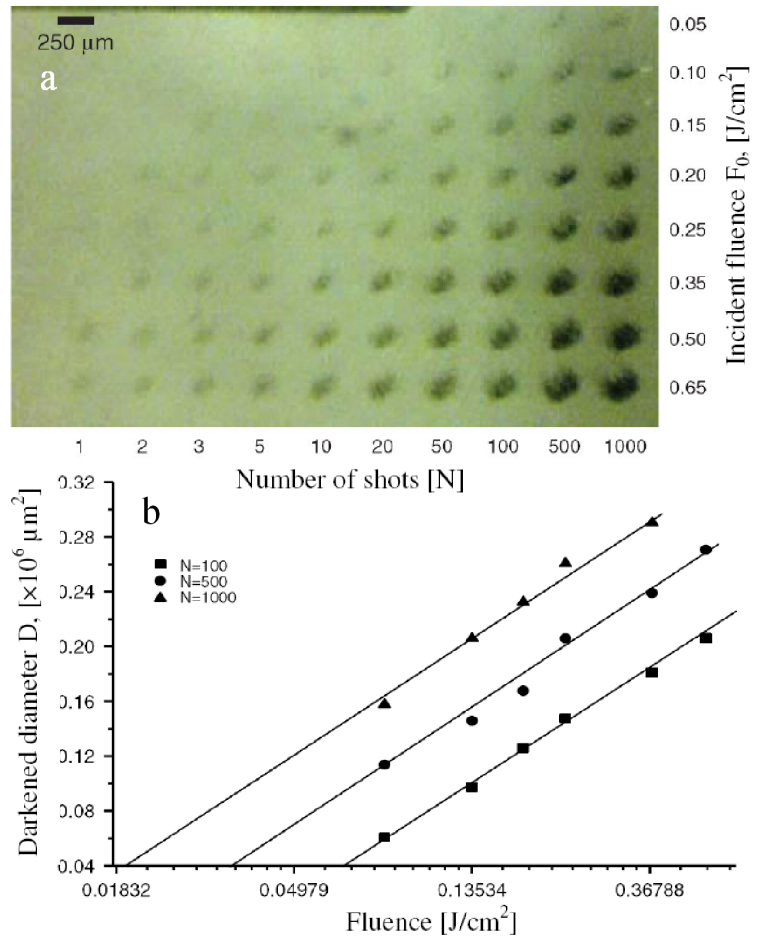

Fig. 3. Optical microscope image of laser induced darkening in $2 \mathrm{~mm}$ SK3 glass sample at different number of laser shots and fluences. (b) The squared diameter, $D^{2}$, of the darkened areas in SK3 as a function of natural logarithm of peak laser fluence, $F_{0}$, at given laser shot numbers of $N=100,500$, and 1000. The solid lines represent the least-squares fit of Eq. (3).

As it is clearly shown in the image (Fig. 3), the area of darkened spots increase with increase of the laser fluence and number of laser shot. In addition, darkening threshold varies at different number of shots. For example, at first row visible darkening is observed after 500 laser shots at fluence level of $0.05 \mathrm{~J} \mathrm{~cm}^{-2}$ while for $0.65 \mathrm{~J} \mathrm{~cm}^{-2}$ laser fluence (latest row), darkening is induced even with applying single laser shot. Annealing of the darkened sample at $150^{\circ} \mathrm{C}$ causes the color centers to disappear and returns the glass sample to its original transparent state without any cracking. This indicates that the induced modifications are not the persistent exchanges.

Increase of darkening diameter with incident laser shots indicates a pulse energy accumulation effect [15]. According to this model, if we consider a Gaussian shaped intensity profile, it becomes clear that the lower intensity, outer edges of the beam, caused no effective modification at low shot numbers for incident laser fluence, but did cause darkening after high number of shots. These results indicate that the darkened areas depend on the applied laser fluence and shot number.
A simple relationship can be derived between the diameter of a darkened area, $D$, and the darkened threshold fluence, $F_{\text {th }}$, at given shot number and the applied laser peak fluence, $F_{0}$. For a Gaussian beam, the spatial fluence profile, $F(r)$, is given by

$$
F(r)=F_{0} \mathrm{e}^{2 r^{2} / \omega^{2}},
$$

where $r$ is the distance from the beam center and $\omega$ is the laser beam spot size. The peak fluence $F_{0}$ and the pulse energy, $E_{\mathrm{p}}$, are directly related by: $F_{0}=2 E_{\mathrm{P}} / \pi \omega^{2}$.

By rearranging of Eq. (2), the diameter $D$ of the modified area and the peak laser fluence $F_{0}$ at the sample satisfy the following relation:

$$
D^{2}=2 \omega^{2} \ln \left(\frac{F_{0}}{F_{\mathrm{th}}}\right) \text {. }
$$

From a plot of the square of the darkened diameters, $D^{2}$, versus the natural logarithm of the applied laser peak fluence $F_{0}$, it is possible to determine the beam radius, $\omega$ and the darkening threshold fluence, $F_{\mathrm{th}}$, for a given applied shot number. Figure $3 \mathrm{~b}$ shows the experimental results for the square of darkened diameters in SK3 glass with respect to the natural logarithm of applied peak fluence at three laser shot numbers of 100,500 , and 1000. The solid linear curves, which are parallel, show theoretical fit to Eq. (3). Intersections of lines with horizontal axes (zero area) represent the darkening threshold fluences and their slop which reflects the effective laser beam spot size was measured to be $160 \pm 5 \mu \mathrm{m}$.

\section{Conclusion}

We have studied, both experimentally and theoretically, the nonlinear optical response in SK3 glass with irradiation by 200 femtosecond laser pulses at $800 \mathrm{~nm}$. A developed theoretical model based on linear, two- and three-photon absorption with including of photogenerated free-carrier absorption effect have used. It is found that the result of presented theory is in good agreement with the experimental results. A fit has allowed extracting the values of three-photon absorption coefficient and photogenerated free-carrier cross-section for the studied SK3 glass sample.

The laser fluence and shot number dependence of laser induced darkening in a $2 \mathrm{~mm}$ thick SK3 glass is illustrated. Several dot fields with different incident pulse energy at different number of shots were generated in glass sample. Darkening thresholds for different laser fluences have been measured. It was found that the darkened threshold depends on the number of incident laser shots. To prediction of experimental values of darkened areas with number of shots and applied laser peak fluence, an accumulation pulse energy model is proposed. Theoretical prediction and experimental results are in good agreement. 


\section{References}

[1] R.L. Sutherland, Handbook of Nonlinear Optics, Marcel Dekker, New York 1996.

[2] M. Yamane, Y. Asahara, Glasses for Photonics, Cambridge University Press, Cambridge U.K. 2000.

[3] L.W. Tutt, T.F. Boggess, Prog. Quantum Electron. 17, 299 (1993).

[4] J. Kruger, W. Kautek, Adv. Polym Sci. 168, 247 (2004), DOI: $10.1007 / \mathrm{b} 12683$.

[5] D. Ashkenasi, K. Jamshidi-Ghaleh, H.J. Hafmann, Photonics West, Proc. SPIE 5339, 16 (2004).

[6] D. Ashkenasi, H.J. Hoffmann, G. Muller, Proc. SPIE 5063, 92 (2003).

[7] C.B. Schaffer, A. Brodeur, J.F. Garc, E. Mazur, Opt. Lett. 26, 93 (2001).

[8] Y. Li, W. Watanabe, K. Yamada, T. Shinagawa, K. Itoh, J. Nishii, Y. Jiang, Appl. Phys. Lett. 80, 1508 (2002).

[9] K. Minoshima, A.M. Kowalevicz, I. Hartl, E.P. Ippen, J.G. Fujimoto, Opt. Lett. 26, 1516 (2001).

[10] R. An, Y. Li, Y.P. Dou, H. Yang, Q.H. Gong, Opt. Express 13, 1855 (2005).
[11] E.N. Glezer, M. Milosavljevic, L. Huang, R.J. Finlay, T.H. Her, J.P. Callan, E. Mazur, Opt. Lett. 21, 2023 (1996).

[12] J.R. Qiu, K. Miura, H. Inouye, J. Nishii, K. Hirao, Nucl. Instrum. Methods B 141, 699 (1998).

[13] O.M. Efimov, K. Gabel, S.V. Garnov, L.B. Glebov, S. Grantham, M. Richardson, M.J. Soileau, J. Opt. Soc. Am. B 15, 193 (1998).

[14] L.A. Siiman, L.B. Glebov, Proc. SPIE 5991, 599112 (2005).

[15] J. Jandeleit, G. Urbasch, H. Hoffmann, H.G. Treusch, E. Kreutz, Appl. Phys. A 63, 117 (1996).

[16] D. Du, X. Liu, G. Mourou, Appl. Phys. B 63, 617 (1996).

[17] K. Jamshidi-Ghaleh, J. Opt. A, Pure Appl. Opt. 11, 1 (2009).

[18] K. Jamshidi-Ghaleh, N. Mansour, D. Ashkenasi, H.J. Hoffmann, Opt. Commun. 246, 213 (2005).

[19] K. Jamshidi-Ghaleh, N. Mansour, A. Namdar, Laser Phys. 15, 1714 (2005).

[20] K. Jamshidi-Ghaleh, N. Mansour, J. Phys. D, Appl. Phys. 40, 366 (2007). 